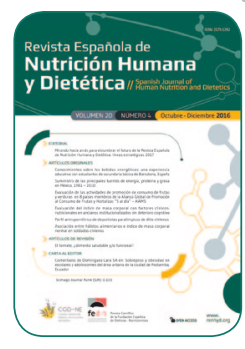

www.renhyd.org

\title{
CARTA AL EDITOR \\ Comentario de Dominguez-Lara SA en: Sobrepeso y obesidad en escolares y adolescentes del área urbana de la ciudad de Riobamba, Ecuador
}

\author{
Sergio Alexis Dominguez-Lara ${ }^{a, *}$

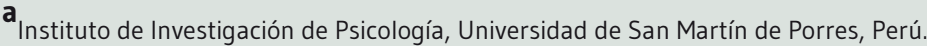 \\ *sdominguezl@usmp.pe
}

Recibido el 10 de febrero de 2016; aceptado el 18 de julio de 2016.

\section{CITA}

Dominguez-Lara SA. Comentario de Dominguez-Lara SA en: Sobrepeso y obesidad en escolares y adolescentes del área urbana de la ciudad de Riobamba, Ecuador. Rev Esp Nutr Hum Diet. 2016; 20(4): 336-37. doi: 10.14306/ renhyd.20.4.210

En el trabajo de Ramos-Padilla y Cols. ${ }^{1}$ se reportan las diferencias entre el exceso de peso, expresado en porcentajes (proporciones), entre diversos grupos (p.e., escolares y adolescentes), y a partir de los análisis estadísticos realizados concluyen que existen diferencias significativas entre los grupos comparados. A pesar de la relevancia de estos resultados en el contexto ecuatoriano, los hallazgos se basan en el test de significancia de la hipótesis nula (NHST, por sus siglas en inglés), es decir, en retener o rechazar una hipótesis nula $\left(\mathrm{H}_{0}\right.$ : no existe diferencia estadísticamente significativa) tomando como referencia el $p$-valor de la prueba estadística en cuestión, es decir, si el $p$-valor es menor que ,05 (o el a elegido), se rechaza $\mathrm{H}_{0}$.

La aplicación de este procedimiento es cuestionada en el análisis de datos en ciencias de la salud por algunos autores, dado que hay pruebas metodológicas que indican que la lógica y los resultados que se derivan de dicho análisis podría ser inadecuado ${ }^{2-4}$, ya que estas pruebas son sensibles al tamaño muestral de los grupos, es decir, tienden a retener la $\mathrm{H}_{0}$ cuando el tamaño muestral es pequeño, y a rechazar $\mathrm{H}_{0}$ cuando es grande.

Actualmente se propone la estimación de la magnitud del efecto (ME) y el uso de intervalos de confianza (IC) para el estadístico en cuestión como procedimientos analíticos complementario al contraste clásico de $\mathrm{H}_{0}$ y que podrían brindar criterios adicionales para interpretar los resultados. En el contexto de la comparación de proporciones, la ME refiere a una forma de cuantificar la magnitud de aquella diferencia que permitan hablar de la importancia práctica de la diferencia encontrada ${ }^{2,3}$. Para el caso que atañe a esta carta, la diferencia de proporciones, el estadístico apropiado para el cálculo ME es la $h$ de Cohen 5 . Su expresión matemática es la siguiente:

$$
\begin{gathered}
h=\phi_{1}-\phi_{2} \\
\Phi=2 \operatorname{arcseno} \sqrt{p}_{p}
\end{gathered}
$$


Donde $\phi$ es la transformación arcseno para la proporción (p) a comparar. La valoración de este estadístico depende de su magnitud y se considera ,20; ,50; y ,80 como ME pequeña, mediana y grande ${ }^{4}$, aunque la valoración dependerá del contexto en el cual es utilizado y no debe considerarse como criterios rígidos. Usualmente, cuando $h$ asume valores cercanos a cero, se interpreta como diferencias triviales o sin importancia práctica para ser interpretadas sustantivamente, es decir, que la diferencia observada entre las proporciones no es lo suficientemente grande para concluir que existen diferencias.

Por otro lado, Ios IC para la diferencia de proporciones $\left({ }^{I C} p_{1}-p_{2}\right)$ hacen referencia al rango de valores que asume el parámetro $\left(P_{1}-P_{2}\right)$ bajo cierto nivel de confianza (usualmente, 95\%), de modo tal que si el IC incluye al cero, es un indicador de que la diferencia entre las proporciones no es estadísticamente significativa ${ }^{6}$, es decir, que las proporciones son estadísticamente similares. Para el cálculo de tales diferencias se recomienda el método Score?

Los resultados en base a los métodos propuestos (Tabla 1) indican que ninguna de las diferencias entre proporciones resulta estadísticamente significativa (IC que contiene el cero), presentando además ME triviales. Esto indica que el peso es estadísticamente similar independientemente de la edad, sexo y tipo de establecimiento al cual asiste la persona. Estos hallazgos difieren de las conclusiones expuestas por Ramos-Padilla y Cols., ya que al basarse enteramente en el NHST, los resultados se orientaron al rechazo de $\mathrm{H}_{0}$ considerando el tamaño muestral utilizado, pero sin tener en cuenta la cercanía de las magnitudes de las proporciones en términos prácticos.

Los métodos expuestos en esta carta pueden ser de utilidad para el investigador en salud, ya que en vista de las limitaciones de la NHST, es necesario contar con alternativas de análisis que tengan supuestos más realistas y que puedan aplicarse a una variedad de circunstancias. Cabe mencionar que existen medidas de ME e IC para todo un amplio rango de análisis estadísticos ${ }^{2,5,8}$, a los que podría acceder el investigador interesado.

\section{CONFLICTO DE INTERESES}

El autor expresa que no hay conflictos de interés al redactar el manuscrito.

\section{REFERENCIAS}

(1) Ramos-Padilla PD, Carpio-Arias TV, Delgado-López VC, Villavicencio-Barriga VD. Sobrepeso y obesidad en escolares y adolescentes del área urbana de la ciudad de Riobamba, Ecuador. Rev Esp Nutr Hum Diet. 2015; 19(1): 21-7.

(2) Fritz CO, Morris PE, Richler J]. Effect size estimates: current use, calculations, and interpretation. J Exp Psychol Gen. 2012; 141(1): 2-18.

(3) Ferguson CJ. An effect size primer: A guide for clinicians and researchers. Prof Psychol Res Pract. 2009; 40(5): 532-8.

(4) Cohen J. A power primer. Psychol Bull. 1992; 112(1): 155-9.

(5) Cohen J. Statistical power analysis for the behavioral sciences. 2a ed. New Jersey: Lawrence Erlbaum Associates; 1988.

(6) Newcombe RG, Merino C. Intervalos de confianza para las estimaciones de proporciones y las diferencias entre ellas. Interdisciplinaria. 2006; 23(2): 141-54.

(7) Newcombe RG. Interval estimation for the difference between independent proportions: comparison of eleven methods. Stat Med. 30 de abril de 1998; 17(8): 873-90.

(8) Grissom RJ, Kim JJ. Effect sizes for research: a broad practical approach. New Jersey: Lawrence Erlbaum Associates; 2005.

Tabla 1. Comparación de proporciones.

Escolares (1) / Adolescentes (2)

Escolares hombres (1) / Escolares mujeres (2)

Establecimientos particulares (1) / Establecimientos fiscales (2)

Adolescentes mujeres (1) / Adolescentes hombres (2)

\begin{tabular}{c:c:c:c}
$\boldsymbol{p}_{1}$ & $\boldsymbol{p}_{2}$ & ${ }^{I C} \boldsymbol{p}_{\boldsymbol{1}}-\boldsymbol{p}_{2}$ & $\boldsymbol{h}$ \\
\hline, 277 &, 215 &,$- 057 ;, 179$ &, 144 \\
, 310 &, 244 &,$- 058 ; 187$ &, 148 \\
, 281 &, 277 &,$- 119 ;, 127$ &, 009 \\
, 233 &, 198 &,$- 079 ; 148$ &, 085
\end{tabular}

p: proporción; $\boldsymbol{h}: \boldsymbol{h}$ de Cohen 